\title{
Saline soil reclamation by means of layered mulch
}

\begin{abstract}
Water conservation and prevention of salt accumulation are important issues in arid and semi-arid regions. Mulching is the most frequently used method for water conservation and prevention of salt accumulation. A mulch with various layers, hence named layered mulch (LM), was applied for reclamation of saline soil. The LM comprises of a light-colored mineral, farmyard manure, and common reed (Arundo donax L.). Two treatments, one with LM and the other without LM (NLM), were carried out on saline soil for 2 years. Soil temperature, water content, and electrical conductivity (EC) were measured during March and September at the depths of 1 to 120 in $4 \mathrm{~cm}$ intervals. The results showed that LM soil had a lower temperature and EC than NLM soils. The LM soil recorded higher soil water content than the NLM soil. The LM soil had a lower temperature possibly because it reflected the sunlight, had high heat capacity, and was less permeable to heat conduction. Consequently, the lower temperature in LM soil resulted in higher soil water content and this can be a soil condition, that enables enhanced salt leaching by subsequent rains.
\end{abstract}

Keyword: Arid region; Salt; Soil water content; Thermal conductivity 